\title{
Centralized Management of Virtual Security Zones in IP Networks
}

\author{
Antti Peltonen, Teemupekka Virtanen, and Esa Turtiainen \\ 1 Ericsson Finland \\ antti.peltonen@ericsson.fi \\ 2 Helsinki University of Technology \\ teemupekka.virtanen@hut.fi \\ 3 Ericsson Finland \\ esa.turtiainen@ericsson.fi
}

\begin{abstract}
The growing security awareness among business users of networks based on Internet Protocol has emerged a need to control the security policies of the network nodes. The nodes can be distributed all over the Internet. The node configuration that is used to enforce the security policy is typically set by hand which is time consuming and error prone. Thus there is a need for centralized management system of the security policies of the nodes.

In this paper we suggest that the roles of network and security administrators should be separated. We have designed a system for centralized security policy management and made a prototype implementation of it. With our system we can control security policies of the nodes securely and remotely from a centralized management node.
\end{abstract}

Keywords: Virtual Security Zones, Security Policy Management

\section{Introduction}

According to Computer Emergency Response Team (CERT) Coordination Center the most common cause for firewall security breaches is misconfigurations [7] and the configuration for the firewall is put in place by the firewall's administrator. Separating the roles of network and security administration would make the situation much simpler as the network administrators no more needed to be aware of security requirements and vice versa.

One of the biggest threats for an enterprise becomes from within the company. Insider attacks are far more common than believed, because companies try to avoid the bad publicity that could follow if the incidents were reported. According to year 2003 CSI/FBI Computer Crime and Security Survey [9] eighty percent of survey respondents had detected insiders abusing the company's network access. By enforcing strong security policies for the hosts in the company's network lowers the possibility for this kind of abuse, along with preventing the flow of highly sensitive data out of the network. 
In order to reach this higher level of protection some additional mechanisms are needed to existing networks. In this study we will identify the enabling technologies and the components that are needed to control security policies of nodes in a public Internet. We will also present a method to separate security management from general system management and divide network into several virtual zones. We have implemented these methods and present the implementation.

\section{Security Policy Enforcement}

Security policy enforcement is the deployment strategy to put the security policy defined by the security administrator in action. The point where the policy decisions are made is called a Policy Decision Point (PDP) [19. The actual enforcement point is called a Policy Enforcement Point (PEP) [19].

Security policy enforcement can be done either in the end-point or in the network. In the end-point based approach the node itself acts as a PEP or there is a dedicated PEP device in place. A good example of the end-point based approach is the distribibuted firewall concept. Another possibility for policy enforcement is to use the network based approach. In this approach there exists a certain node in the network that the end-point needs to contact before being able to contact the desired host. In other words the PEP resides at the end-point, while the PDP is somewhere in the network.

We noticed that policy enforcement approaches can be divided by using two parameters - is the approach dependent on the network topology and does the approach use strong authentication. With strong authentication we mean that the hosts trust each other explicitly. Trust relationship can be established for example by using PKI or web of trust. In weak authentication hosts base their trust directly to other host's identifier that may be for example an IP address or a host name. Based on this categorization we have identified one approach that fits into every class. Our results are presented in Table 1.

Table 1. Approaches to policy enforcement

\begin{tabular}{|c|c|c|}
\hline & Weak authentication & Strong authentication \\
\hline Topology dependent & Conventional firewall & $\begin{array}{c}\text { Security clearance server } \\
\text { with firewall }\end{array}$ \\
\hline Topology independent & $\begin{array}{c}\text { Host identity } \\
\text { protocol }\end{array}$ & Distributed firewall \\
\hline
\end{tabular}

\section{Existing Administration Tools and Current Technology}

The SNMPv3 provides authorization, authentication and confidentiality protection [17, thus being the recommended version for all network management applications. The data items for different devices are specified in the Management 
Information Base (MIB) specifications. MacFaden et al. discuss the configuration of networks using the SNMP protocol in [14]. This document represents the best practices for designing MIB modules, implementation of SNMP configuration agents, and discusses deployment and security issues as well.

Extensible Markup Language (XML) [18] is a language for describing flexible common information formats and the related data. XML may be used to present almost anything, including also security policies. The DAXFi project [1] has devised a dynamic XML firewall that uses XML to specify the firewall security policy. By using XML, the security policies can be defined in a vendor independent manner, and then later translated to vendor specific commands. We see this approach as one of the best solutions for describing security policies in multivendor environments.

An interesting approach to firewall policy definition is presented by Bartal et al. in their paper about Firmato a novel firewall management toolkit [3]. The Firmato constitutes of a Model Definition Language (MDL), Entity-Relationship $(\mathrm{E} / \mathrm{R})$ model, a model compiler and a visualization component. The basic concept in Firmato is a role. Roles define the capabilities of initiating and accepting services. We think that the abstraction layer that the Firmato brings into firewall rule definition is a huge step forward in firewall management. The security administrator is no more obliged to do the tedious configuration work using low level configuration files, but instead use a modelling language.

Conventional firewalls depend on the topology of the network. If a host is moved beyond the firewall perimeter the policy enforcement does not apply anymore. The PEP and PDP both reside in the firewall and the policy decisions are based on preconfigured filter lists.

In order to have a topology independent approach that uses strong authentication an approach like distributed firewall may be applied. In distributed firewall the PEP is located at the host, while the PDP may be located at the same place or somewhere else in the network. The concept of distributed firewall was first introduced by Steven M. Bellovin in his paper Distributed Firewalls [4].

Host Identity Protocol (HIP) 15] is an attempt to break the binding between the host's identity and its location. This is achieved by introducing a new cryptographic name space and protocol layer between the transport and network layers. In HIP each end-point has a distinguishing Host Identifier (HI). In order to communicate between a pair of end-points the initiating end-point must learn one of the IP addresses the other end-point is associated to. This is achieved using an address resolution service.

KeyNote [6] [5] is a trust management system that provides a language with the same name for defining policy rules conveniently. The purpose of trustmanagement system is to provide a standard mechanism for specifying application security policies and credentials. [6]

In our opinion the concept of a trust-management system is quite easily applicable to networking, too. An important point is that the trust management should be done on stream not on packet basis. On packet basis this would consume too much processing time. However, every stream passing a compliance 
checker once would be no problem. Hedbom et al. noticed that the security of a firewall or Intrusion Detection System (IDS) itself is very important [12].

Virtual Private Network (VPN) is a very widely and wildly used concept. It is used in several different contexts to mean different things. In this paper we will understand the VPN as a way to transfer private data traffic using a public network without exposing it to public. VPNs provide a more cost effective way to provide private networking for multi-site communication than traditional approaches such as leased lines. Tunneling is one of the principles used in VPN networks to carry traffic over the IP backbone network. 10] Tunneling is especially useful when the payload traffic have no relation to underlying IP addressing. This is the case when the payload traffic is multiprotocol or private IP addressing [16] is in use.

\section{Virtual Security Zones}

\subsection{Design}

The general level architecture for the system is depicted in the Figure 1. The central component of the system is the security policy server, which is the server hosting the software component for managing the virtual security zones. In close connection to the security policy server work the directory servers, which are used as distribution points for security policy definition files. In addition to these, a certificate authority is used for assigning certificates for the security policy server and managed nodes. These certificates act as digital identities of their owners. PKI was chosen for the trust establishment system as it provides the best scalability and availability.

We have also a natural hierarchy in our system as every node needs to trust the central point - the security policy server. Mutual authentication between the security policy server and the managed nodes is achieved using CA assigned certificates. In the managed node, a small program is needed to fetch the security policy definition files from one of the directory servers, and interpret the acquired file into executable commands.

Local administrator is the person responsible for local administrative tasks on the node. These tasks include hardware and software installation and configuration of the node. The local administrator also initiates the certificate enrollment for acquiring the certificate for the node. Network administrator is the person responsible for configuring the general network infrastructure. He will assign the IP addresses that the nodes will use either manually or automatically using the DHCP protocol. If the node is supposed to be part of a virtual security zone, the network administrator will pass the information about the node to the security policy server.

Security administrator is the person responsible for setting up the security zones. He is also responsible for administration of the network administrators' access rights to the central management node. Security administrator trusts that the certificates assigned by the certificate authority are valid.

Certificate Authority is the entity who assigns the digital certificates for security policy server and managed nodes. It will act as a trusted third party 
between the security policy server and the nodes. In the case that security policy server and the managed nodes are below different PKIs a cross certification process 2 is needed to establish the trust between the two PKIs.

Security Policy Manager (SPM) is the software component for the centralized management of the security policies in the security zones. The component is located in the security policy server. It will assemble the security policy configuration files for managed nodes and delegate the distribution of these files to the directory servers.

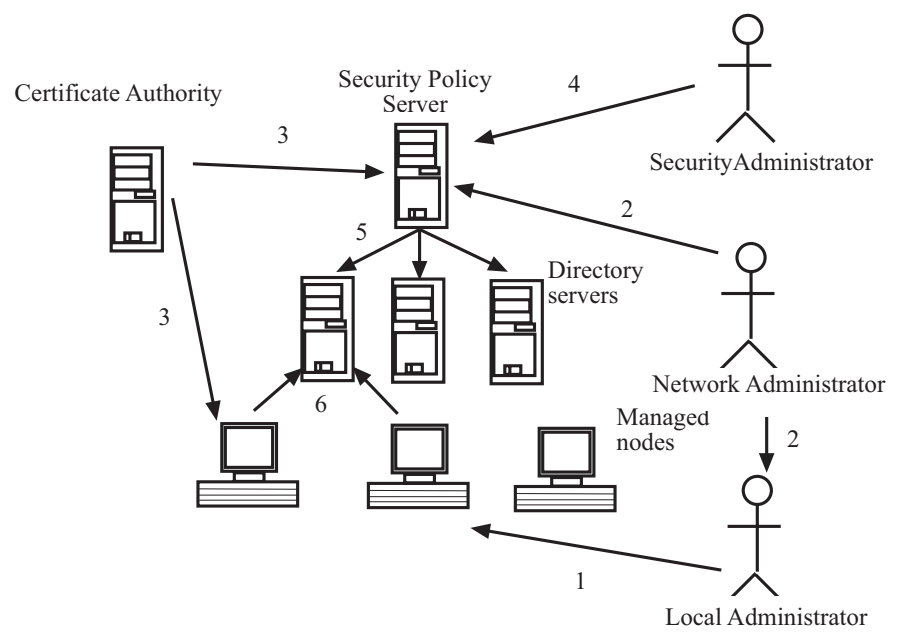

Fig. 1. General architecture for the system

Security Policy Agent (SPA) is the software component installed in the managed nodes for retrieving and processing the configuration information written by the SPM. The SPA together with firewall and VPN software acts as a software based policy enforcement point (PEP).

Directory Servers act as distribution points for security policy configuration files. Managed nodes will contact one of the directory servers to acquire their own configuration.

Managed nodes form security zones according to security policies defined by the security policy administrator. Managed nodes listen for policy updates from security policy server and fetch their configuration files from directory servers.

\subsection{Policy Transfer}

The policy decision can be made either in the central management node or at the managed PEP. However, the former approach is unreasonable in our case. Because the policy decisions are made per packet basis, the traffic towards the central management node would become outrageously high. We therefore chose 
to combine the PDP with the PEP. This way the security policy information needs to be transferred to the managed node only during system startup and when the security policy is changed.

Based on the literature study we examined the different possibilities for transferring the policy from central management node to the PEP. The most promising methods we identified were using SNMP for policy transfer, or using XML or configuration commands to describe the policy, and other means to transfer it. We wanted to make the presentation of the policy independent from the transfer process, which then left us with the last two choices. If the policy is mapped to low level commands already at the SPM, the SPM needs to exactly know the target environment. However, often this information is not easily available or it would require extra work from one of the administrators to figure it out. Therefore we chose to use the XML language as the high level policy description language, which is used in the policy transfer. The XML based message is then mapped to environment specific low level configuration commands at the PEP in the managed node.

The Document Type Definition (DTD) for the XML file used for policy transfer is depicted in Figure 2. The root element, the policy, can have any number of ipsec groups (ipsec group) in it. The ipsec groups consists of a preshared key (psk) and any number of end-points (end point). End-points always have an IP address (remote ip) and they may have a tunneling configuration (tunnel). If a tunneling configuration is present the tunnel element will include the virtual IP address of the remote node (remote vip) and a corresponding virtual IP address for the local node (own vip). The tunneling can be used to create an extra logical layer on top of the public IP network.

We used a similar process to that used in PGP [62] to sign and encrypt the configuration information. Data is first compressed to enhance the resistance against cryptanalysis [21].

To be able to notify the managed node about a change in the security policy two mechanisms can be used - server push or client pull. In our system the configuration changes do not occur often, therefore the server push is the preferred method, as it creates less network traffic compared to client pull. However, the server push generates a heavy load on the security policy server if the number of clients in the security zone is high. Therefore we chose to use a slightly modified server push approach.

\footnotetext{
$<$ ? xml version=" 1.0 " encoding="utf- 8 "? $>$ $<$ !-- DTD forsecurity policy presentation --> $<$ !ELEMENT policy(ipsec_group*) $>$

$<$ !ELEMENT ipsec_group(psk, end_point*) $>$

$<$ !ELEMENT psk(\#PCDATA)>

$<$ !ELEMENT end point(remote ip, tunnel?)>

$<$ !ELEMENT remote_ip(\#PCDĀTA) $>$

$<$ !ELEMENT tunnel(remote vip, own vip) $>$

$<$ !ELEMENT remote vip(\#PCDATA $)>$

$<$ !ELEMENT own_vìp(\#PCDATA) $>$
}

Fig. 2. The DTD for policy presentation 
When the security policy is changed the server sends a short notification message to the client. This will initiate the client side update process. The listening daemon process in the managed node - the SPA - wakes up and starts the configuration process for the node.

\subsection{Security Policy Manager}

Security Policy Manager (SPM) is the software component that is used for managing the security policies of the distributed managed nodes. The SPM process is hosted by the security policy server. We will next formulate the requirements for the SPM in the form of use cases.

The primary actors using services of the system are administrators and nodes. The administrator actor is extended with two special types of administrator network administrator and security administrator. Every administrator needs to login into the system before being able to do any other type of action.

The network administrator has a use case for inserting node into system and for removing node from the system. Using these two functions the Network administrator can join and remove nodes from the group of managed nodes.

The security administrator has use cases for creating, modifying and deleting security zones. The create zone allows security administrator to define new security zone and its parameters. The parameters include the name of the zone, the shared secret the nodes in the zone will use for mutual authentication, and optionally the private address space to be used in the zone. The modify zone is used for adding or removing nodes from an existing security zone, and the delete zone removes the whole security zone. All these three use cases use the notify node use case for notifying managed nodes about configuration changes.

Only one use case was defined for managed node actor. This is called get configuration. It allows the managed node to retrieve its configuration information from the SPM. The storage of configuration files need not be at the same server the SPM is located, but the task can be delegated for directory servers as we depicted earlier in the system architecture.

When considering a node inside a security zone, certain information about these entities need to be maintained. We begin by modeling the node. The managed node has a name that is used by the network and security administrators to identify it. It has also an unique IP-address that can be used for distinguishing the nodes from each other. The node can also be a member of any number of security zones. A security zone has a distinguished name that is used for identifying it. In addition, a shared secret that the nodes use for identifying members of the zone is required. Optionally the nodes may communicate using private address space instead of the public IP addresses. Therefore also the private address space, or virtual address space as we call it, is stored in the database. The security zone can have any number of nodes in it. 


\subsection{Security Policy Agent}

Security Policy Agent is the software component located in the managed nodes. It is responsible for retrieving configuration files from SPM and putting the received security policy in action.

The configuration update of the client consists of the following phases: (1) Node downloads the compressed and encrypted configuration file, content encryption key and signature of the configuration file from one of the directory servers. (2) Node decrypts the content encryption key with its private key. (3) Node decrypts the configuration file with the content encryption key. (4) Node verifies the signature of the decrypted configuration file. (5) Node decompresses the configuration file. (6) Node maps the XML based policy contained in the configuration file to environment specific commands. (7) Node executes the commands.

After executing all the aforementioned steps the node will be running with the new configuration. If there is a problem in any step, the configuration update will fail and the program will return to its initial state. One reason for a failed configuration update could be a network failure.

\subsection{Security Policy Enforcement}

Instead of presenting another high level policy definition language (see [8] [5]) we took a hands- on approach to solving the problem of security policy management in distributed network. We will include in our prototype implementation the support for configuring firewall and VPN policies.

Although any protocol capable of traffic encryption could be used, we chose the IPSec for encrypting the traffic in the virtual security zones. The IPSec being a part of the forthcoming IPv6 standard, will most probably have a strong position in encrypting future network traffic, not forgetting its already wide use in the $\mathrm{IPV}_{\mathrm{V}}$ networks. We also wanted to combine the VPNs with the distributed firewall concept to provide a totally isolated VPN or virtual security zone.

A comprehensive security solution can not depend solely on a packet filtering firewall although the firewall can be a crucial part of it. To secure a node we need to control several different things. We have listed some of the most important ones in the following list: user or program access rights, filtering incoming and leaving traffic, encrypting the leaving traffic, user authentication and authorization, and intrusion detection

Firewall's purpose is to enforce the security policy defined by the security administrator. It accomplishes this by filtering the traffic at packet, stream or application level. According to Ziegler in Linux Firewalls [20], a packet filtering firewall can protect you against the following threats: some source address spoofing, useful information revealed in response to port scan, malformed broadcast packets used to identify UNIX systems, some forms of network mapping, some denial-of-service attacks, source-routed packets, some forms of fragmentation bombs, local mistakes that affect remote sites, access to private Local Area Network (LAN) services, and additional protection against local server misconfigurations 
However, as we pointed out already in the introductory chapter, a firewall can only be as good as its administrator is. In a centralized remote configuration, the hopefully highly competent security administrator can verify that the configuration is really error free before distributing the configuration to managed nodes. Centralized administration also makes it easy to change the configuration very rapidly throughout the network if a flaw is found.

\subsection{Communication Model between the Managed Nodes}

We wanted to let the managed nodes communicate using a private address space, while on the other hand we wanted to provide a secure communication channel over the public network. Analysis of the different VPN technologies lead us to the combination of two of these - namely GRE with IPSec. GRE provides the tunneling of the private addresses, while IPSec handles the data encryption and integrity protection.

Using this approach we can form logically separate networks from the underlying public IP address space. Only the virtual IP-address is provided for applications, thus making those independent from the underlying public IPaddress. In other words, we will use the public IP-addresses of the nodes just for packet transmission and decapsulate the virtual IP-address at the receiving node. Combining the end point firewalls to this structure will lead us to the construction of a virtual security zone.

\section{Implementation}

J2EE was chosen as the server implementation environment because it supports the Java servlet and Java Server Pages (JSP) technologies, and thus enables easy development of web frontends.

Java servlets are server side components that handle the client requests in an efficient and highly versatile way. JSPs are used to create the presentation front-end for the data that the servlets provide. JSP files are interpreted by the Apache Tomcat to Hypertext Markup Language (HTML) pages that can be shown by the client in a web browser. The actual data is stored inside a relational database system. Apache Ant [1] is a build tool for Java, developed in the Apache Jakarta Project. It uses feature rich XML based configuration language to describe the build and deployment process. All build scripts for our project were written using the scripting language of the Ant.

\subsection{Software Components}

In Figure 3 we have depicted the classes for the SPM server and how they are divided into packages. The web package consists of the two servlet classes which handle the requests made by the security and network administrators from their web browsers. These classes work in close connection with the classes in the util package. 


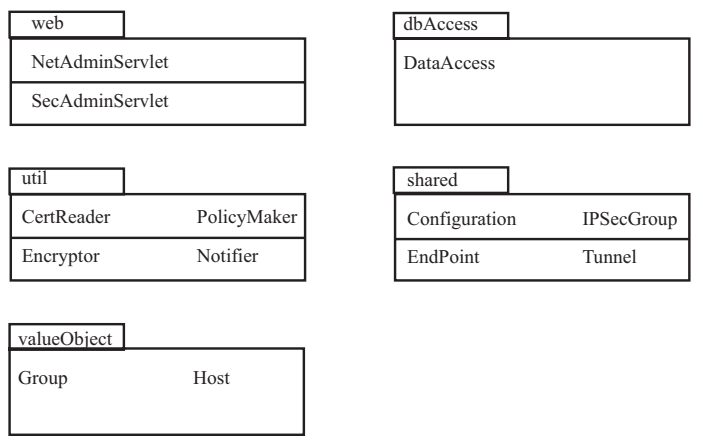

Fig. 3. Classes and packages for Security Policy Manager

The util package includes most of the advanced functionality embedded into the system. In advance to having the actual logic for forming the XML based configuration files for managed nodes (PolicyMaker), it includes worker classes for reading certificates from keystore (CertReader), encrypting data (Encryptor) and notifying the client hosts about configuration changes (Notifier). The data acquired from database is handled using the classes in the valueObject package.

Configuration data is saved into object structure which corresponds to the XML presentation we have introduced. The corresponding classes are located in the shared package, which as the name indicates, is shared between the SPM and SPA applications. A configuration may have any number of IPSec groups and each IPSec group may have any number of end-points. End-points can have a tunnel dependency if the private IP addressing is in use. Data persistence is handled by the DataAccess class in the dbAccess package. It provides methods for storing, querying and deleting data. The actual database access is naturally done using SQL language.

\subsection{User Interface for Administrators}

The Security Policy Manager provides a convenient web-based user interface for network and security administrators. Being web-based, the interface is available everywhere there is a web-browser. The connection between the web-browser and the server is protected with TLS/SSL and the administrators are authenticated using username and password.

The security administrator can conveniently select the nodes he wants in the zone by clicking the radio box next to the hostname and IP address. He also needs to define a name for the zone and a shared secret that the zone members will use to authenticate each other. This secret should probably be generated automatically by the system because the administrator is likely to choose easy and too short shared secrets. The last field allows the administrator to give a virtual network address space for the chosen managed nodes. 


\subsection{Security Policy Agent}

The Security Policy Agent (SPA) is the daemon process running in the nodes we want to manage remotely. It includes functionality for retrieving, processing and installing the configuration data.

The SPA has three packages - core, shared and util. In the core package is the main program class for the client called SPAgent. It is responsible for reading client specific configuration from initialization file, retrieving the initial configuration from one of the directory servers, and for starting the process listening for incoming configuration updates.

\section{Conclusions}

In this study we constructed a system for centralized management of virtual security zones. The choice for policy transfer from centralized management node to the managed nodes was an XML based policy file. The integrity and confidentiality of the file was protected using strong cryptography. The actual policy enforcement was done by the policy enforcement point in the managed node. To prevent users and local administrator from changing the security policy the security administrator has set, the policy enforcement point should be tamper resistant.

We also presented a new concept called a virtual security zone. The members of the virtual security zone are isolated from the underlying public IP network using tunneling and encryption. In our implementation the traffic inside the virtual security zone was encrypted using the IPSec protocol. Applications running on the nodes can be separated into different zones using virtual IP addresses provided by the GRE tunneling.

The management of security policies in a distributed environment has traditionally been a task including lot of handwork. By handwork we mean that the configurations of the hosts are either locally or remotely set up using a command line interface. Our system provides an easy way to define virtual security zones that can span hosts in multiple mutually untrusted networks in a centralized manner. The configuration is done securely by using strong cryptography to provide confidentiality as well as integrity protection for the configuration data. We also provided a web based management front-end for the administrators, that made the security administration independent from time and place.

One interesting area for further research would be the support for the mobility of the managed hosts. While our system could provide limited mobility with slight modifications, further research in the area would be needed to enable the managed hosts move freely while still maintaining the security policy the security administrator had set in the first place. 


\section{References}

1. DAXFi - Dynamic XML Firewall http://daxfi.sourceforge.net

2. C. Adams and S. Farrel: Internet X.509 Public Key Infrastructure Certificate Management Protocols. IETF RFC 2510. March 1999.

3. Yair Bartal, Alain Mayer, Kobbi Nissim, and Avishal Wool: A Novel Firewall Management Toolkit, in Proceedings of the 20th IEEE Symposium on Security and Privacy, 1999.

4. Steven Bellovin: Distributed firewalls, ;login: 24(Security), November 1999.

5. M. Blaze, J. Feigenbaum, J. Ioannidis, and A. Keromytis The KeyNote TrustManagement System Version 2. IETF RFC 2704. September 1999.

6. Matt Blaze, Joan Feigenbaum, A. Angelos, and D. Keromytis KeyNote: Trust management for public-key infrastructures, in Proceedings of the Cambridge 1998 Security Protocols International Workshop, 1998.

7. CERT. Install firewall hardware and software. http://www.cert.org/securityimprovement/ practices/p056.html

8. Nicodemos Damianou, Naranker Dulay, Emil Lupu, Morris Sloman. The Ponder Policy Specification Language, in Proceedings of Workshop on Policies for Distributed Systems and Networks (Policy2001), 2001.

9. Robert Richardson (Editorial Director). Eighth Annual CSI/FBI Computer Crime and Security Survey. 2003. Available at: http://www.gocsi.com

10. Paul Ferguson, Geoff Huston. What is a VPN, The Internet Protocol Journal 1(2), 1998.

11. Apache Software Foundation. Apache Ant. Available at: http://ant.apache.org

12. Hans Hedbom, H Kan Kvarnstr M, Erland Jonsson, A Protection Scheme for Security Policies Using One-Way Functions, tech.rep. 01-3, Chalmers University of Technology, 2001.

13. S. Kent, R. Atkinson. Security Architecture for the Internet Protocol. IETF RFC 2401. November 1998.

14. M. Macfaden, D. Partain, J. Saperia, W. Tackabury. Configuring Networks and Devices with Simple Network Management Protocol (SNMP). IETF RFC 3512. April 2003.

15. Pekka Nikander, Jukka Ylitalo, Jorma Wall. Integrating Security, Mobility, and Multi-Homing in a HIP Way, in Proceedings of Network and Distributed Systems Security Symposium (NDSS'03), page 87-99, Internet Society, San Diego, CA, Feb 2003.

16. Y. Rekhter, B. Moskowitz, D. Karenberg, G. J. De Groot, E. Lear. Address Allocation for Private Internets. IETF RFC 1918. February 1996.

17. William Stallings. SNMPv3: A Security Enhancement for SNMP, IEEE Communications Surveys \& Tutorials 1 (Fourth Quarter), 1998. Available at: http://www.comsoc.org/livepubs/surveys/public/4q98issue/stallings.html

18. World Wide Web Consortium (W3C). Extensible Markup Language (XML). Available at: http://www.w3c.org/XML

19. R. Yavatkar, D. Pendarakis, R. Guerin. A Framework for Policy-based Admission Control. IETF RFC 2753. January 2000.

20. Robert L. Ziegler. Linux Firewalls, second edition, New Riders Publishing, 2002.

21. P. R. Zimmermann. The Official PGP User's Guide, MIT Press, 1995. 